\title{
Gestational and postnatal modulation of esophageal sphincter reflexes in human premature neonates
}

\author{
Sudarshan R. Jadcherla1-3, Theresa R. Shubert ${ }^{1}$, Manish B. Malkar ${ }^{1-3}$, Swetha Sitaram ${ }^{1}$, Rebecca K. Moore ${ }^{1,2}$, Lai Wei ${ }^{4}$, \\ Soledad Fernandez $z^{4}$ and Robert G. Castile ${ }^{1,2,5}$
}

BACKGROUND: Effects of gestational age (GA) and postnatal maturation on upper and lower esophageal sphincter (UES and LES) reflex development remain unclear. We hypothesized very-preterm (VPT) born neonates ( $<32$ wk GA) have delayed maturation of UES contractile reflex (UESCR) and LES relaxation reflex (LESRR) vs. preterm (PT) born (32-37 wk GA) neonates.

METHODS: Using provocative manometry, effects of 1,263 graded mid-esophageal stimuli (air, liquid) on sensory-motor characteristics of UESCR and LESRR were investigated in 24 VPT-born and 12 PT-born neonates (37.8 \pm 0.6 vs. $38.9 \pm 0.4 \mathrm{wk}$ postmenstrual age respectively, $P=0.14$ ).

RESULTS: In response to liquid stimuli (vs. air), VPT-born neonates displayed prolonged UESCR and LESRR response latencies $(P<0.001)$ and prolonged UESCR and LESRR durations $(P<0.01)$; unlike PT-born neonates, who exhibit prolonged LESRR response latency $(P<0.01)$, but similar UESCR and LESRR durations $(P=0.2)$. Differences were noted in LESRR duration in VPT Vs. PT neonates for air stimuli $(P=0.04)$. With liquid stimuli, increasing GA was associated with decreasing response onset latencies to UESCR and $\operatorname{LESRR}(P<0.05)$, and increasing LESRR duration ( $P=0.02)$.

CONCLUSION: Using GA as categorical or continuous variable, vagus-mediated mechano-sensitive and liquid-sensitive reflex characteristics of UESCR and LESRR are distinct; LESRR differs with varying intrauterine maturation suggesting inhibitory modulation progresses with advancing maturation.

G lobally, between 12.3 and 18.1 million premature babies (< 37 wk gestational age (GA)) are born annually (1). In the United States, of approximately 4 million births annually, about $12 \%$ are premature (2). Prematurely born infants experience growth failure as well as delays in achieving feeding milestones, thus contributing to prolonged hospitalization (3). Oral feeding difficulties become pronounced around term equivalent postmenstrual age (PMA), commonly manifesting as feeding-related aerodigestive symptoms, gastroesophageal reflux
(GER), and oropharyngeal or esophageal dysphagia (3-6). Infant dysphagia, if not properly evaluated and managed, can lead to prolonged tube feeding, development of oral aversion, and impaired quality of life as the infant matures, persisting as dysfunctional feeding behavior. The precise etiologies of infant dysphagia are multifaceted; however premature birth is a known circumstance from which this condition precipitates, in addition to lung disease, prolonged length of hospital stay, and exposure to Neonatal Intensive Care Unit (NICU) interventions. In gestationally immature very-preterm (VPT) infants, maturation of oromotor skills ex utero (as opposed to in utero maturation with their gestationally older counterparts) may have an unknown effect on the development of physiological components which facilitate the development of safe oral feeding skills $(3,7,8)$.

Recent developments have allowed the use of micromanometric evaluation of aerodigestive reflexes in preterm infants (9-12), as well as the longitudinal assessment of these neuromotor markers relevant to the esophageal sphincters and esophageal body (13-16). The upper esophageal sphincter (UES), a conglomeration of skeletal muscles, and the lower esophageal sphincter (LES) constituted by highly specialized smooth muscle (17-20), provide important barrier functions for airway protection and esophageal clearance during swallowing and GER events. Specifically, aerodigestive protective reflexes such as UES contraction safeguards against retrograde GER ascent, and LES relaxation facilitates luminal clearance during peristalsis. Central and enteric nervous system interactions advance around 32-34 wk GA or PMA, manifesting as oropharyngeal, gastrointestinal sphincter, and peristaltic reflexes, or as cue-based feeding skills (13-16,21-26). In infants born preterm, aerodigestive adaptive reflexes develop under a different set of sensory exposure and environmental conditions, compared with infants who have developed these skills while in utero. Comparison of these characteristics within the preterm group with varying gestational immaturity and development under the NICU conditions remains unclear. The clarification of this gap in knowledge is important

${ }^{1}$ The Neonatal and Infant Feeding Disorders Program, Center for Perinatal Research, The Research Institute at Nationwide Children's Hospital, Columbus, Ohio; ${ }^{2}$ The Neonatal Aerodigestive \& Pulmonary Program, Center for Perinatal Research, The Research Institute at Nationwide Children's Hospital, Columbus, Ohio; ${ }^{3}$ Divisions of Neonatology, Pediatric Gastroenterology and Nutrition, Department of Pediatrics, The Ohio State University College of Medicine, Columbus, Ohio; ${ }^{4}$ Center for Biostatistics, The Ohio State University College of Medicine, Columbus, Ohio; ${ }^{5}$ Division of Respiratory Medicine, Department of Pediatrics, The Ohio State University College of Medicine, Columbus, Ohio. Correspondence: Sudarshan R. Jadcherla (sudarshan.jadcherla@nationwidechildrens.org) 
in understanding the pathophysiological basis of aerodigestive symptoms and evolving neurocircuitry that are of relevance in more immature preterm infants.

Therefore, our aims were to evaluate the vagally-mediated adaptive reflexes participating in aero-digestive tract protection across different maturational states of in utero development, with the hypothesis that the VPT-born group has delayed maturation of UES contractile reflex (UESCR) and LES relaxation reflex (LESRR) characteristics when compared to the gestationally older preterm (PT)-born group, but studied at similar term equivalent PMA.

\section{RESULTS}

\section{Demographic and Clinical Characteristics}

Twenty-four VPT-born $(1.1 \pm 0.4 \mathrm{~kg}$ birth weight, $2.4 \pm 0.7$ weight at study) and 12 PT-born $(2.6 \pm 0.9 \mathrm{~kg}$ birth weight, $3.0 \pm 1$ weight at study) infants born at $28.1 \pm 2.1$ vs. $35.2 \pm 1.7$ wk GA respectively $(P<0.001)$ were investigated at $37.8 \pm 2.8$ vs. $38.9 \pm 1.5$ wk PMA respectively $(P=0.14)$. Data from 1,263 esophageal responses $(n=837$ in VPT-born; $n$ $=426$ in PT-born) to stimuli were analyzed. Within the first week of life, intraventricular hemorrhage was noted among five infants in the VPT-born group (two infants with grade 1 , and one each with grades 2,3 , or 4 ) vs. three infants in PT-born group who all had grade 1 intraventricular hemorrhage ( $P=0.78$ for group comparisons). Clinical characteristics regarding the duration of aerodigestive and pulmonary support for infants are stated in Table 1. At manometric evaluation, among 24 VPT-born infants, 50\% were on exclusive full oral feeds and 50\% were transitioning to full oral feeds while among 12 PT-born infants, $66.7 \%$ were receiving exclusive full oral feeds and $33.3 \%$ were transitioning to full oral feeds $(P=$ 0.34 for group comparison).

\section{Characteristics of Stimulus-Induced UESCR}

A representative response to an esophageal infusion with the analytical parameters labeled is shown in Figure 1. Specific to UESCR, the frequency of responses for both groups increased to both stimuli as the volumes increased $(P<0.0001$, Table 2$)$.

Media grouped analysis indicated prolonged UESCR response latency to liquid (vs. air) infusions in VPT-born infants $(P<0.001)$, but not PT-born infants (Table 3$)$. Active

Table 1. Duration of aerodigestive support

\begin{tabular}{lrcc}
\hline Clinical characteristics & $\begin{array}{c}\text { Very } \\
\text { preterm }\end{array}$ & Preterm & $P$ value \\
\hline Days of NICU exposure @ TOS & $69 \pm 22$ & $26 \pm 13$ & $<0.0001$ \\
Days of ventilation & $12.4 \pm 4.3$ & $1.7 \pm 0.8$ & 0.008 \\
Days of NCPAP & $21.5 \pm 4.1$ & $1.2 \pm 0.6$ & 0.0001 \\
Days of O & $35.3 \pm 6.0$ & $6.6 \pm 3.5$ & 0.005 \\
Total days of gavage feeding & $40.8 \pm 5.3$ & $3.9 \pm 2.4$ & $<0.0001$ \\
Days of caffeine use & $24.3 \pm 4.3$ & $5.7 \pm 4.7$ & 0.006 \\
Duration of overall NICU stay, days & $79 \pm 36$ & $32 \pm 24$ & 0.004 \\
\hline TTime of study; data presented as mean \pm SD. & & &
\end{tabular}

UESCR duration was also prolonged for liquid (vs. air) for VPTborn infants $(P=0.003)$, but not for PT-born infants (Table 3$)$. Group comparisons between VPT-born and PT-born infants regarding response latency and active UESCR duration were similar for both media.

When GA at birth was considered as a continuous variable, while controlling for PMA and stimulus volume, we found that UESCR response latency to liquid infusions decreased $(P$ $<0.01$ ) with increasing birth gestation; however this effect was not seen with air stimuli (Table 4). Duration of active UESCR (Table 4) was not significantly associated with GA in response to either liquid or air provocation.

\section{Characteristics of Stimulus-Induced LESRR}

A representative response to an esophageal infusion with the analysis parameters labeled is shown in Figure 1. Specific to LESRR, the frequency response for both groups increased for both stimuli as the volumes increased $(P<0.0001$, Table 2$)$ except for PT-born group in response to air infusions.

Media grouped analysis indicated prolonged LESRR response latency to liquid (vs. air) in both VPT-born $(P<$ $0.001)$ and PT-born $(P=0.004)$ infants (Table 3$)$. Duration of active LESRR was prolonged in response to liquid (vs. air) in VPT-born infants $(P=0.006$; Table 3$)$, but not for PT-born infants $(P=0.17)$. LESRR nadir duration was similar between air vs. liquid stimuli for both the VPT-born group ( $3.6 \pm 0.5 \mathrm{vs}$. $3.8 \pm 0.7$ respectively, $P=0.7)$, and the PT-born group $(3.9 \pm 0.7$ vs. $4.1 \pm 0.8$ respectively, $P=0.91$ ). Group comparisons between VPT-born and PT-born infants regarding LESRR response latency and nadir duration were similar for either medium. However, VPT-born infants had a significantly shorter duration of active LES relaxation compared with PT-born infants in response to air infusions $(P=0.04)$ but not to liquid infusions (Table 3).

In response to liquid infusions, but not for air, while controlling for different volumes as well as PMA using a mixed linear regression model, increasing GA was associated with decreasing response latency to LESRR onset (Table 4; $P=0.04$ ) and increasing duration of active LESRR (Table 4; $P=0.02$ ).

\section{DISCUSSION}

Gestational immaturity and biological variability coupled with the development of aerodigestive protective reflexes under the NICU conditions remains unclear. In this study, our goal was to clarify the sensory-motor characteristics of the UESCR and the LESRR representative of vagal excitatory and inhibitory drive respectively. Specifically, our aims were to evaluate the vagally-mediated adaptive reflexes participating in aerodigestive tract protection across different maturational states of in utero and ex utero development, with the hypothesis that the VPT-born group has delayed maturation of UESCR and LESRR characteristics when compared to the gestationally older PT-born group, when studied at similar term equivalent PMA.

The cardinal findings and plausible physiological explanation of our study findings pertinent to UESCR are as follows: 


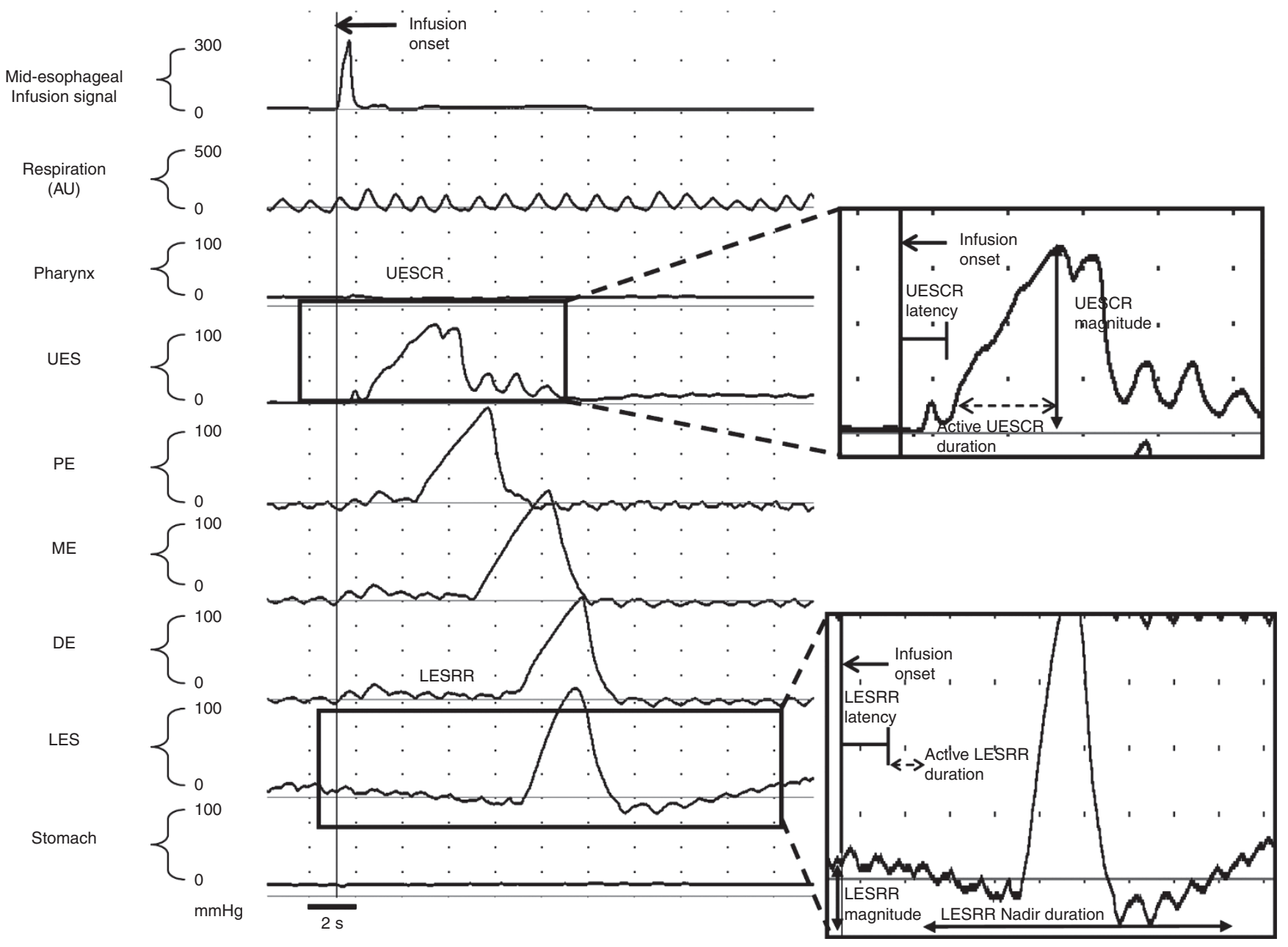

Figure 1. Representation of manometric reflexive responses to a mid-esophageal infusion. LESRR, lower esophageal sphincter relaxation reflex; UESCR, upper esophageal sphincter contractile reflex. Note: Labeled parameters used for analysis, including response latency, magnitude, and duration characteristics of both UESCR and LESRR.

Table 2. Frequency of reflex recruitment increases with volume of infusion

\begin{tabular}{llcc}
\hline Air & Group & Odds ratio & Pvalue \\
\hline UESCR & VPT & $4.0(2.3-7.0)$ & $<\mathbf{0 . 0 0 0 1}$ \\
& PT & $3.9(2.6-6.0)$ & $<\mathbf{0 . 0 0 0 1}$ \\
LESRR & VPT & $2.4(1.7-3.4)$ & $<\mathbf{0 . 0 0 0 1}$ \\
& PT & $1.6(1.0-2.6)$ & 0.06
\end{tabular}

Liquid

UESCR

$$
\text { VPT }
$$

PT

LESRR

$$
\text { VPT }
$$$$
3.2(2.2-4.7)
$$$$
2.8(2.0-4.1)
$$$$
\text { PT }
$$$$
2.2(1.6-3.0)
$$

$<0.0001$

$<0.0001$

$<0.0001$

$<0.0001$

With each unit of increasing infusion volume, the odds of reflex recruitment for both UESCR and LESRR increase, with the exception of PT response to air infusions. Data presented as OR (95\% confidence interval). Bolded values indicate significant $P$ values. LESRR, lower esophageal sphincter relaxation reflex; VPT, very-preterm; PT, preterm; UESCR, upper esophageal sphincter contractile reflex.

(i) When studied at term equivalent PMA, VPT-born infants have similarities approaching that of the PT-born group in regards to response latency and response duration of UESCR during mechanosensitive (air) or liquid-sensitive stimulation. These findings may be due to similarities with the development of afferent and efferent excitatory pathways modulating UES contractile functions, and maybe indicators of neuroplasticity in the VPT group. These findings also indicate that, at similar PMA, VPT and PT infants have similar ability to recruit airway protective mechanisms. (ii) While analyzing the effect of birth gestation as a continuous variable and controlling for liquid stimulus volumes as well as the PMA at evaluation, we found that response latency to UESCR shortens with increasing birth gestation. Therefore, it is likely that sensory-motor effects for handling liquids improve with increasing gestation. This may be related to prolonged experience of swallowing amniotic fluid, or shorter NICU duration prior to introduction of oral feeds. This finding also indicates that with increasing gestational immaturity at birth, comes prolonged initiation of airway protective reflexes in the form of UESCR. When initiation of airway protection is delayed, the infant becomes susceptible to aspiration. Aspiration is a contraindication to safe oral feeding, thus leading to delayed progression toward reaching this milestone. 
Table 3. Sensory motor characteristics of UESCR and LESRR

\begin{tabular}{|c|c|c|c|c|}
\hline & $\begin{array}{c}\text { Response } \\
\text { characteristics }\end{array}$ & Air & Liquid & $P$ value \\
\hline \multirow[t]{2}{*}{ Very Preterm } & $\begin{array}{l}\text { Response latency } \\
\text { to UESCR, seconds }\end{array}$ & $2.9 \pm 0.3$ & $4.7 \pm 0.4$ & $<0.001$ \\
\hline & $\begin{array}{l}\text { Duration of active } \\
\text { UESCR, seconds }\end{array}$ & $2.7 \pm 0.3$ & $3.3 \pm 0.2$ & 0.003 \\
\hline \multirow[t]{2}{*}{ Preterm } & $\begin{array}{l}\text { Response latency } \\
\text { to UESCR, seconds }\end{array}$ & $3.7 \pm 0.4$ & $3.8 \pm 0.6$ & 0.08 \\
\hline & $\begin{array}{l}\text { Duration of active } \\
\text { UESCR, seconds }\end{array}$ & $2.8 \pm 0.3$ & $2.9 \pm 0.3$ & 0.56 \\
\hline \multirow[t]{2}{*}{ Very Preterm } & $\begin{array}{l}\text { Response latency } \\
\text { to LESRR, seconds }\end{array}$ & $3.1 \pm 0.2$ & $4.7 \pm 0.5$ & $<0.001$ \\
\hline & $\begin{array}{l}\text { Duration of active } \\
\text { LESRR, seconds }\end{array}$ & $2.0 \pm 0.4^{*}$ & $2.5 \pm 0.4$ & 0.006 \\
\hline \multirow[t]{2}{*}{ Preterm } & $\begin{array}{l}\text { Response latency } \\
\text { to LESRR, seconds }\end{array}$ & $2.7 \pm 0.3$ & $4.0 \pm 0.7$ & 0.004 \\
\hline & $\begin{array}{l}\text { Duration of active } \\
\text { LESRR, seconds }\end{array}$ & $3.8 \pm 0.7$ & $3.8 \pm 0.5$ & 0.17 \\
\hline
\end{tabular}

Data presented as mean \pm SE. ${ }^{*} P<0.05$ (vs. preterm). Bolded values indicate significant Pvalues.

LESRR, lower esophageal sphincter relaxation reflex; UESCR, upper esophageal sphincter contractile reflex.

The cardinal findings and plausible physiological explanation of these findings pertinent to LESRR are as follows: (i) Duration of active LESRR in the VPT group was prolonged with liquids (vs. air stimulus), while this was not the case in the PT group. This fact suggests that each group distinguishes gas and liquid esophageal provocation differently. Air stimuli have the property of either rapid mechanosensitive distention or upstream spread or inducing a belch to expel air out. On the other hand, liquid stimuli need to be swallowed back during peristalsis. Additionally, the time necessary for the LES to achieve full relaxation is increased when VPT infants are handling a liquid bolus, thus the ability of the infant to fully clear liquid material may not be as effective as the ability of the infant to clear air. (ii) VPT born infants have a significantly shorter duration of active LES relaxation with air when compared with PT-born infants (with prolonged active LES relaxation); this was not so for liquids. This may be an indication that VPT infants are in a state of hypervigilance when an air bolus is present. On the other hand, as the LES becomes fully relaxed more rapidly in VPT infants, when compared with PT infants, they may become more susceptible to material refluxing from the stomach. (iii) Finally, considering increasing GA as a continuous variable while controlling for stimulus media, stimulus volumes and PMA at evaluation, we noted decreasing response latency to LESRR, as well as prolonged duration of active LESRR, with liquid stimuli. This fact suggests that with liquid esophageal stimulation (but not with air), there is modulation (increase in inhibitory and decrease in excitatory) of LES tone, which is likely to contribute to our findings, i.e., faster response latency to LES relaxation and prolonged duration of active LESRR. These findings indicate that as infants spend more time in utero, in response to a liquid bolus, as would occur in the presence of oral feeding or during
Table 4. Effect of gestational age at birth on UESCR and LESRR characteristics

\begin{tabular}{|c|c|c|c|c|}
\hline \multirow[b]{2}{*}{ Characteristics } & \multicolumn{2}{|l|}{ air } & \multicolumn{2}{|c|}{ liquid } \\
\hline & Estimate $\pm \mathrm{SE}$ & $P$ value & Estimate $\pm \mathrm{SE}$ & $P$ value \\
\hline \multicolumn{5}{|l|}{ UESCR characteristics } \\
\hline $\begin{array}{l}\text { Response latency to } \\
\text { UESCR onset, seconds }\end{array}$ & $0.04 \pm 0.05$ & 0.45 & $-0.18 \pm 0.1$ & 0.01 \\
\hline $\begin{array}{l}\text { Duration of active } \\
\text { UESCR, seconds }\end{array}$ & $0.1 \pm 0.1$ & 0.10 & $-0.03 \pm 0.05$ & 0.5 \\
\hline \multicolumn{5}{|l|}{ LESRR characteristics } \\
\hline $\begin{array}{l}\text { Response latency to } \\
\text { LESRR, seconds }\end{array}$ & $-0.06 \pm 0.05$ & 0.25 & $-0.14 \pm 0.1$ & 0.04 \\
\hline $\begin{array}{l}\text { Duration of active } \\
\text { LESRR, seconds }\end{array}$ & $0.12 \pm 0.08$ & 0.15 & $0.18 \pm 0.1$ & 0.02 \\
\hline
\end{tabular}

liquid GER clearance, the onset of LES relaxation begins more quickly and occurs over a longer duration, in order to optimize timing of complete LES relaxation to facilitate clearance of the bolus into the stomach.

The functional neural connectivity and neural networks regulating UESCR and LESRR have been characterized $(15,16,27-29)$. The differences in proximal and distal esophageal sphincter sensory-motor characteristics in VPT-born and PT-born infants are suggestive of variations in modulated vagal excitatory and/or inhibitory neurons when the infant is maturing ex utero. Homo sapiens are not born with a completely myelinated functioning vagal system, however, it has been previously reported that starting at $24 \mathrm{wk}$ GA, there is a rapid increase in the total number of myelinated vagal fibers, with the greatest increase beginning at 30-32 wk GA $(30,31)$. In the event of birth before this developmental window, as is the case with more premature GA, neural myelination is an aspect which could be modulated, therefore contributing to modulated sensory-motor reflexive responses. Additionally, recent studies support that UES contractility and respiratory rhythms and aberrations are closely interconnected (32). Therefore, prolonged UESCR latency and active duration in VPT-born infants may modify and prolong respiratory adjustments, specifically with regard to oral feeding.

Myelination is not the sole element of the nervous system which undergoes continued maturation after the transition from fetus to neonate. While certain motor patterns are present in the gastrointestinal tract and continue to mature during fetal development, differentiation of enteric nerve cells continues through the first years of life (33). After premature birth, this highly plastic neural network is exposed to the NICU environment as it continues to develop. Involving the current cohort, VPT-born infants required a greater degree of aerodigestive support after birth in the form of endotracheal tubes and chronic indwelling gavage feeding tubes, and had experienced a longer NICU stay at the time of study, which can be a stressful situation for infants. The link between stress and inflammation of the gastrointestinal system in adults 
is widely reported (34-36). It is possible that VPT neonates under duress experience a similar inflammatory response in their gastrointestinal tract. Such an effect may be lessened in infants born closer to term.

Secondary peristalsis can be initiated at the level of either the striated muscle esophagus or the smooth muscle esophagus and is associated with LES relaxation $(11,16)$. Mid-esophageal mechano-stimulation results in more esophago-deglutition responses in infants at earlier stages of maturation (as in VPTborn infants) (21). There is debate over whether the mediation of the LES is central or peripheral; however there is strong evidence suggesting that the central nervous system is the primary regulator of esophago-deglutition responses, while secondary peristalsis can be mediated locally (37-39). Because LESRR associated with esophago-deglutition responses is centrally modulated, as opposed to local modulation as in the case of LESRR associated with secondary peristalsis, the active duration of LESRR is decreased for VPT infants in response to air infusion as the central neural signal transmission is more rapid. In response to liquid stimulation, degree of immaturity at birth resulted in prolonged response latency to UESCR and LESRR, thus delaying activation of airway protective mechanisms (UESCR) leading to prolonged presence of material in the esophagus (delayed LESRR as well). Unless other hierarchical adaptive reflexes participate in the protection of the aerodigestive tract $(15,27,28,32)$, this situation increases vulnerability to aspiration in VPT infants. With increased gestational maturation, as in PT infants, nicotinic or muscarinic excitatory or inhibitory regulation may be modulated differently (compared with greater duration of ex utero maturation as in VPT infants) resulting in variation with motor responses between the groups.

In PT-born infants, there was no increase in the frequency of LESRR in response to increasing infusion volumes of air. The discrepancy between air and liquid stimulus induced effects in PT-born infants was not evident in VPT-born infants, and may be related to environmental exposure of infants born earlier. Additionally, the in utero fetus clears amniotic fluid via pharyngeal swallowing activity $(40,41)$,. The nuclei responsible for control of smooth and skeletal muscle reflex responses use this process to adapt with those pathways; adaptation of which may differ in infants born earlier.

Like any clinical study, our study has limitations. The population is heterogeneous within the clustered groups of VPTborn or PT-born infants. As our goals were to recognize the differences and similarities in healthy VPT-born infants vs. PT-born infants at similar, near full term PMA, we chose the single stratification point of $32 \mathrm{wk}$ based on pragmatic, scientific, and clinical appropriateness. It is difficult to recruit and study healthy full-term born neonates for ethical and pragmatic reasons. Hence, the current PT-born cohort satisfies the need for a comparison group (for VPT-born neonates), as both groups were transitioning to oral feeding at the time of evaluation. Additionally, unexplained effects as a result of care provided to preterm infants from birth to hospital discharge require further investigation.
Clinical and future research implications are as follows: (i) Careful evaluation of reasons for elective induction for premature deliveries before $39 \mathrm{wk}$ may be beneficial. Linear regression results indicate that with every additional week of gestation the fetus further develops important aerodigestive reflexes through what is considered early term pregnancy ( $37 \mathrm{wk})$. Additionally, infants born at $37 \mathrm{wk}$ GA have a 7.5 -fold increased rate of respiratory distress syndrome than those born at $39 \mathrm{wk}$ (42). These data suggest that promoting a 39 -wk gestation period allows the fetus to properly develop airway and digestive function. This supports statements from American Congress of Obstetricians and Gynecologists $(43,44)$. (ii) Provision of adequate nutrition is critical to the development of neuromuscular tissue and organs in premature infants, and allowing for proper adaptation of the enteric nervous system to life outside the uterus. The role and quantity of specific nutrients in myelination, muscle growth, and development of inhibitory modulation is not well understood. We speculate that adequate supplementation of essential amino acids and fats may result in the optimal development of excitatory and inhibitory neuromuscular patterns. Our study approach can be helpful in characterizing vagal excitatory and inhibitory regulation during maturation. (iii) Clearly, the PT infant group had decreased exposure to aerodigestive interventions. We speculate that minimizing aerodigestive tract interventions by limiting them to critical needs, combined with those interventions that promote swallowing, may decrease the contribution of aversive stimuli and favor deglutition, thus modifying sensitivities and neural adaptation.

In conclusion, UES and LES aerodigestive protective reflexes induced upon esophageal provocation undergo maturation with GA regarding afferent and efferent signal transmission and processing of excitatory and inhibitory effects on the sphincters. These changes in sphincter function characterize the influence of external factors versus continued gestational maturation on the adaptation of the enteric nervous system as part of the transition from fetal to neonatal life. This study provides a mechanistic reasoning behind delays in achievement of aerodigestive milestones after premature birth, and forms a basis to investigate chronic aerodigestive illnesses in prematurely born infants.

\section{METHODS \\ Subjects}

Participants were 36 premature infants classified into VPT-born (born $<32$ wk GA), and PT-born (born $\geq 32$ wk GA), per March of Dimes classification (2). Infant GA was determined from maternal obstetric records, and PMA was calculated by adding chronological age to GA. None of the subjects were receiving prokinetics, acid suppressive therapy, or xanthines at study or at discharge. Subjects with perinatal asphyxia and congenital birth defects were excluded. Subjects were in various stages of transition from gavage to oral feeds.

The study procedures and protocols were approved by the ethics committee at Institutional Research Review Board (IRB) at Nationwide Children's Hospital Research Institute, Columbus, Ohio, USA. This study protocol adheres to the guidelines set forth in the IRB policy in addition to the health insurance portability and accountability acts. Informed consent and health insurance portability and accountability acts authorization were obtained from parents or legal guardians prior to study. 


\section{Esophageal Manometry Methods}

Manometry methods have been adopted as previously described by us $(11,13,14,21)$. Briefly, the manometry catheter assembly comprised of dual sleeves to record the UES and LES, and four side ports to record motility at the pharynx, proximal-, middle-, and distal esophageal segments, in addition to a terminal gastric recording port. The catheter assembly (Dentsleeve International, Mui Scientific, Ontario, Canada) was connected to the pneumohydraulic micromanometric water perfusion and recording systems via the resistors, pressure transducers (TNF-R disposable pressure transducers, MMS medical instruments, Dover, NH) and amplifiers (Solar modules, Solar-2, MMS medical instruments, Dover, $\mathrm{NH}$ ). The micromanometric water perfusion rates were $0.04 \mathrm{ml} / \mathrm{min}$ /port for the UES and LES sleeve sensors $(11,13-15,21)$.

\section{Manometric Experimental Protocol}

The catheter was calibrated at the level of the infant's esophagus, and an infant-size appropriate manometry catheter was passed nasally with the infant being unsedated and lying supine. The catheter was properly positioned using the pull through technique to identify the high pressure zones defining the UES and LES, and was well secured. After allowing infants to adapt to catheter placement for about 15$20 \mathrm{~min}$, infusion protocol was begun. Data were continuously acquired and analyses were performed $(13,14,21)$. To test UES and LES reflex responses, we recorded upstream and downstream responses to graded volumes of abrupt mid-esophageal infusions of air (mechanosensitive stimulus) and liquids (liquid sensitive stimulus) administered in triplicate $(0.1,0.5,1.0,2.0$, and $5.0 \mathrm{ml}$ respectively for air; $0.1,0.5,1.0$, and $2.0 \mathrm{ml}$ respectively for liquids). All infants underwent this infusion protocol and data were subsequently analyzed.

\section{Data Analysis and A Priori Analytical Definitions of Neuromotor} Markers of Reflexes

Manometric waveform analysis was performed as previously described $(13-16,21)$. Manometry data analysis was done by a trained investigator who was blinded to group (VPT or PT) assignment. Briefly, resting pressures in both the upper and lower esophageal sphincters were taken at least $5 \mathrm{~s}$ before any particular infusion during esophageal and respiratory quiescence. UESCR was defined as an increase of at least $4 \mathrm{mmHg}$ from baseline within $12 \mathrm{~s}$ from the stimulus onset. UESCR latency was defined as the duration from the onset of stimulus to this initial $4 \mathrm{mmHg}$ increase in UES pressure. Active UESCR duration was defined as the time from this initial $4 \mathrm{mmHg}$ increase to the maximum contraction amplitude. UESCR magnitude was defined as the differential pressure between basal UES and maximum contraction amplitude. LESRR onset was defined as a decrease of at least $5 \mathrm{mmHg}$ below LES resting pressure. LESRR latency was defined as the duration from infusion onset to this initial $5 \mathrm{mmHg}$ decrease in LES pressure. Active LESRR duration was defined as the time from the initial drop in $5 \mathrm{mmHg}$ from resting tone to the nadir pressure. LESRR magnitude was defined as the pressure difference between basal LES pressure and LES nadir pressure. LESRR nadir duration was defined as the duration from LES pressure drop to $5.0 \mathrm{mmHg}$ to the time of LES pressure recovery to $5.0 \mathrm{mmHg}$. All measurements were taken at end expiration.

\section{Statistical Analysis}

Chi squared and unpaired $t$-tests were used to evaluate clinical characteristics between the groups at time of study and at discharge (Table 1). Demographic and clinical data are reported as mean \pm SD. Manometric data are reported as mean \pm SE, unless otherwise stated. Reflexes were visually defined at the time of study. Due to the presence of repeated measures, GEE models (with repeated statement) were performed for categorical responses (presence of UESCR and LESRR) while controlling for different infusion volumes, GA and PMA. Mixed models were used to make comparisons between media (air vs. liquid) and groups (PT vs. VPT) while controlling for PMA and infusion volumes (Table 2). Similarly, mixed models were used for continuous responses while controlling for different infusion volumes and PMA (Table 3 ). Since GA and PMA were poorly correlated (Pearson's $r=0.30, P<0.0001$ ) and were not collinear (variance inflation factor $=1.15$ ), they were included in the same regression model
(45). $P$ values $<0.05$ were considered statistically significant. Analyses were conducted using SAS version 9.3 (SAS Institute, Cary, NC).

\section{ACKNOWLEDGMENT}

The authors thank Juan Peng and Vanessa Parks for statistical and technical support.

\section{STATEMENT OF FINANCIAL SUPPORT}

This study was supported in part by the National Institutes of Health (Bethesda, MD), R01 DK 068158 (Jadcherla) and P01 DK 068051 (Jadcherla/ Shaker).

Disclosure: The authors have no real or perceived conflicts of interest to declare.

\section{REFERENCES}

1. Blencowe H, Cousens S, Oestergaard MZ, et al. National, regional, and worldwide estimates of preterm birth rates in the year 2010 with time trends since 1990 for selected countries: a systematic analysis and implications. Lancet 2012;379:2162-72.

2. March of Dimes. March of Dimes 2012 Premature Birth Report Card, 2012. https://www.marchofdimes.com/peristats/pdflib/998/US.pdf.

3. Jadcherla SR, Wang M, Vijayapal AS, Leuthner SR. Impact of prematurity and co-morbidities on feeding milestones in neonates: a retrospective study. J Perinatol 2010;30:201-8.

4. American Academy of Pediatrics Committee on Fetus and Newborn. Hospital discharge of the high-risk neonate. Pediatrics 2008;122:1119-26.

5. Barlow SM, Estep M. Central pattern generation and the motor infrastructure for suck, respiration, and speech. J Commun Disord 2006;39:366-80.

6. Lau C, Smith EO, Schanler RJ. Coordination of suck-swallow and swallow respiration in preterm infants. Acta Paediatr 2003;92:721-7.

7. Jadcherla SR, Stoner E, Gupta A, et al. Evaluation and management of neonatal dysphagia: impact of pharyngoesophageal motility studies and multidisciplinary feeding strategy. J Pediatr Gastroenterol Nutr 2009;48: 186-92.

8. Jadcherla SR, Peng J, Moore R, et al. Impact of personalized feeding program in 100 NICU infants: pathophysiology-based approach for better outcomes. J Pediatr Gastroenterol Nutr 2012;54:62-70.

9. Staiano A, Boccia G, Salvia G, Zappulli D, Clouse RE. Development of esophageal peristalsis in preterm and term neonates. Gastroenterology 2007;132:1718-25.

10. Omari TI, Miki K, Fraser R, et al. Esophageal body and lower esophageal sphincter function in healthy premature infants. Gastroenterology 1995;109:1757-64.

11. Jadcherla SR. Manometric evaluation of esophageal-protective reflexes in infants and children. Am J Med 2003;115 Suppl 3A:157S-60S.

12. Omari TI, Benninga MA, Barnett CP, Haslam RR, Davidson GP, Dent J. Characterization of esophageal body and lower esophageal sphincter motor function in the very premature neonate. J Pediatr 1999;135:517-21.

13. Jadcherla SR, Duong HQ, Hoffmann RG, Shaker R. Esophageal body and upper esophageal sphincter motor responses to esophageal provocation during maturation in preterm newborns. J Pediatr 2003;143:31-8.

14. Jadcherla SR, Duong HQ, Hofmann C, Hoffmann R, Shaker R. Characteristics of upper oesophageal sphincter and oesophageal body during maturation in healthy human neonates compared with adults. Neurogastroenterol Motil 2005;17:663-70.

15. Gupta A, Gulati P, Kim W, Fernandez S, Shaker R, Jadcherla SR. Effect of postnatal maturation on the mechanisms of esophageal propulsion in preterm human neonates: primary and secondary peristalsis. Am J Gastroenterol 2009;104:411-9.

16. Pena EM, Parks VN, Peng J, et al. Lower esophageal sphincter relaxation reflex kinetics: effects of peristaltic reflexes and maturation in human premature neonates. Am J Physiol Gastrointest Liver Physiol 2010;299: G1386-95.

17. Bitar KN. Function of gastrointestinal smooth muscle: from signaling to contractile proteins. Am J Med 2003;115 Suppl 3A:15S-23S.

18. Biancani P, Sohn UD, Rich HG, Harnett KM, Behar J. Signal transduction pathways in esophageal and lower esophageal sphincter circular muscle. Am J Med 1997;103(5A):23S-8S. 
19. Mittal RK, Balaban DH. The esophagogastric junction. N Engl J Med 1997;336:924-32.

20. Biancani P, Goyal RK, Phillips A, Spiro HM. Mechanics of sphincter action. Studies on the lower esophageal sphincter. J Clin Invest 1973;52:2973-8.

21. Jadcherla SR, Hoffmann RG, Shaker R. Effect of maturation of the magnitude of mechanosensitive and chemosensitive reflexes in the premature human esophagus. J Pediatr 2006;149:77-82.

22. Gupta A, Jadcherla SR. The relationship between somatic growth and in vivo esophageal segmental and sphincteric growth in human neonates. J Pediatr Gastroenterol Nutr 2006;43:35-41.

23. Jadcherla SR, Berseth CL. Effect of erythromycin on gastroduodenal contractile activity in developing neonates. J Pediatr Gastroenterol Nutr 2002;34:16-22.

24. Amaizu N, Shulman R, Schanler R, Lau C. Maturation of oral feeding skills in preterm infants. Acta Paediatr 2008;97:61-7.

25. Ittmann PI, Amarnath R, Berseth CL. Maturation of antroduodenal motor activity in preterm and term infants. Dig Dis Sci 1992;37:14-9.

26. Davidson E, Hinton D, Ryan-Wenger N, Jadcherla S. Quality improvement study of effectiveness of cue-based feeding in infants with bronchopulmonary dysplasia in the neonatal intensive care unit. J Obstet Gynecol Neonatal Nurs 2013;42:629-40.

27. Jadcherla SR, Gupta A, Coley BD, Fernandez S, Shaker R. Esophago-glottal closure reflex in human infants: a novel reflex elicited with concurrent manometry and ultrasonography. Am J Gastroenterol 2007;102:2286-93.

28. Jadcherla SR, Gupta A, Wang M, Coley BD, Fernandez S, Shaker R. Definition and implications of novel pharyngo-glottal reflex in human infants using concurrent manometry ultrasonography. Am J Gastroenterol 2009;104:2572-82.

29. Jean A. Brain stem control of swallowing: neuronal network and cellular mechanisms. Physiol Rev 2001;81:929-69.

30. Sachis PN, Armstrong DL, Becker LE, Bryan AC. Myelination of the human vagus nerve from 24 weeks postconceptional age to adolescence. J Neuropathol Exp Neurol 1982;41:466-72.

31. Porges SW, Furman SA. The Early Development of the Autonomic Nervous System Provides a Neural Platform for Social Behavior: A Polyvagal Perspective. Infant Child Dev 2011;20:106-18.
32. Hasenstab KA, Jadcherla SR. Respiratory events in infants presenting with apparent life threatening events: is there an explanation from esophageal motility? J Pediatr 2014;165:250-255.e1.

33. Puri P, Rolle U. Development of the enteric nervous system. In: Holschneider AM, Puri P, eds. Hirschsprung's Disease and Allied Disorders. Springer Berlin: Heidelberg, 2008. pp. 13-20.

34. Mayer EA. The neurobiology of stress and gastrointestinal disease. Gut 2000;47:861-9.

35. Vishnar A, Ghulam R, Mittal RK. Non ulcer dyspepsia and its correlation with life stress, anxiety and depression. Indian J Psychiatry 2000;42:88-93.

36. Goyal RK, Hirano I. The enteric nervous system. N Engl J Med 1996;334:1106-15.

37. Hendrix TR. Coordination of peristalsis in pharynx and esophagus. Dysphagia 1993;8:74-8.

38. Holloway RH, Dodds WJ, Helm JF, Hogan WJ, Dent J, Arndorfer RC. Integrity of cholinergic innervation to the lower esophageal sphincter in achalasia. Gastroenterology 1986;90:924-9.

39. Gyawali CP, Bredenoord AJ, Conklin JL, et al. Evaluation of esophageal motor function in clinical practice. Neurogastroenterol Motil 2013;25: 99-133.

40. Grassi R, Farina R, Floriani I, Amodio F, Romano S. Assessment of fetal swallowing with gray-scale and color Doppler sonography. AJR Am J Roentgenol 2005;185:1322-7.

41. Miller JL, Sonies BC, Macedonia C. Emergence of oropharyngeal, laryngeal and swallowing activity in the developing fetal upper aerodigestive tract: an ultrasound evaluation. Early Hum Dev 2003;71:61-87.

42. Engle WA, Kominiarek MA. Late preterm infants, early term infants, and timing of elective deliveries. Clin Perinatol 2008;35:325-41, vi.

43. American College of Obstetricians \& Gynecologists. ACOG committee opinion no. 561: Nonmedically indicated early-term deliveries. Obstet Gynecol 2013;121:911-5.

44. Poets CF, Wallwiener D, Vetter K. Risks associated with delivering infants 2 to 6 weeks before term-a review of recent data. Dtsch Arztebl Int 2012;109:721-6.

45. Montgomery DC, Peck EA. Vining Introduction to Linear Regression Analysis. 3rd edn. New York: Jon Wiley and Sons, 2001. 